\title{
Near-infrared spatial solitons in heavy metal oxide glasses
}

\author{
A. Pasquazi, ${ }^{1}$ S. Stivala, ${ }^{1}$ G. Assanto, ${ }^{1, *}$ J. Gonzalo, $^{2}$ J. Solis $^{2}{ }^{\text {and C. N. }}$ Afonso ${ }^{2}$ \\ ${ }^{1}$ NooEL-Nonlinear Optics and OptoElectronics Lab, Consorzio Nazionale Interuniversitario per le Scienze Fisiche \\ della Materia, Istituto Nazionale di Fisica Nucleare, and Department of Electronic Engineering-University "Roma Tre," \\ Via della Vasca Navale 84, 00146 Rome, Italy \\ ${ }^{2}$ Laser Processing Group, Instituto de Optica, Consejo Superior de Investigaciones Científicas, Serrano 121, \\ 28006 Madrid, Spain \\ *Corresponding author: assanto@uniroma3.it
}

Received March 23, 2007; revised May 17, 2007; accepted May 18, 2007; posted May 30, 2007 (Doc. ID 81414); published July 19, 2007

We demonstrate two-dimensional spatial solitons excited by near-infrared picosecond pulses in Kerr-like heavy metal oxide glasses with a nonlinearity one order of magnitude larger than in fused silica. Solitons were obtained at $820 \mathrm{~nm}$ owing to the presence of multiphoton absorption, which prevented catastrophic collapse. (c) 2007 Optical Society of America

OCIS codes: $190.5940,190.4400$.

Bright optical spatial solitons are self-trapped beams that result from the balance between diffraction and self-focusing [1]. They are ubiquitous and have been observed in media with various physical properties [2-5]. At variance with the case of waveguides with nonlinearity acting in one transverse dimension, spatial solitons due to an intensity-dependent response in bulk tend to undergo catastrophic beam collapse unless additional effects intervene to arrest it. Among such stabilizing phenomena are nonlocality (i.e., a response extending beyond the excitation), index saturation, ionization, and nonlinear absorption [3-6]. For these reasons and in spite of the excellent chemical and dielectric properties of glasses (including stability, low losses, extended transmittance, and fast nonlinearity) [7-9], spatial solitons in glass have been reported in a few cases: with femtosecond pulsed beams in planar waveguides $[10,11]$, in the presence of multiphoton ionization and damage $[12,13]$, with $\mathrm{cw}$ excitation in self-focusing thermooptic [13], and in photorefractive systems [14]. More recently, experiments on filaments generated by color-center formation through multiphoton ionization in fused silica using fs ultraviolet pulses were reported [15]. Particularly interesting within the scenario of glasslike nonlinear amorphous materials are compound oxides with a large content of heavy metal ions. They exhibit phonon energies considerably lower than in silicate and borate systems with an optical transparency interval extending beyond $7-8 \mu \mathrm{m}$ and large linear $(>2)$ and ultrafast Kerr refractive indices [7,8,17-19] that, in conjunction with multiphoton absorption, could support stable $(2+1) \mathrm{D}$ optical solitonlike beams [11,20].

In this Letter we demonstrate Kerr-based nondestructive optical self-confinement of light beams over six Rayleigh lengths in a novel, high-index, heavy metal oxide glass of the ternary system $\mathrm{Nb}_{2} \mathrm{O}_{5}-\mathrm{PbO}-\mathrm{GeO}_{2}$ (NPG) [21,22]. By comparing the nonlinear responses at 1.064 and $0.82 \mu \mathrm{m}$, we show that picosecond excitation of 2D spatial solitons (or "solitary waves" owing to absorption) can be achieved in a stable fashion by virtue of three-photon absorption (3PA), which saturates self-focusing at $820 \mathrm{~nm}$, i.e., in the first spectral window for fiber optics communication. Moreover, by numerically fitting the experimental data, we were able to evaluate both the Kerr and the 3PA coefficients of NPG at $820 \mathrm{~nm}$.

NPG glasses with nominal composition 25 $\mathrm{Nb}_{2} \mathrm{O}_{5}-25 \mathrm{PbO}-50 \mathrm{GeO}_{2}$ mol.\% were prepared by mixing high-purity reagents: $\mathrm{PbO}(99.9995 \%), \mathrm{Nb}_{2} \mathrm{O}_{5}$ (99.999\%), and $\mathrm{GeO}_{2}(99.999 \%)$. The mixture was melted in a platinum crucible, in a furnace at $1100-1300^{\circ} \mathrm{C}$ for $1 \mathrm{~h}$ and then poured onto a brass plate before annealing at $450^{\circ} \mathrm{C}$ for $1 \mathrm{~h}$. Finally, the transparent yellowish glass was cooled to room temperature at $1.5^{\circ} \mathrm{C} / \mathrm{min}$. Refractive index and transmission were determined by spectroscopic ellipsometry, as shown in Fig. 1 [21]. Degenerate four-wave mixing at $800 \mathrm{~nm}$ provided a nonlinear refractive index $n_{2} \sim 10^{-15}-10^{-14} \mathrm{~cm}^{2} / \mathrm{W}$.

Propagation experiments were carried out in a sample of $5.7 \mathrm{~mm} \times 8.7 \mathrm{~mm} \times 11.5 \mathrm{~mm}$ at two wavelengths with low linear absorption, namely 820 and $1064 \mathrm{~nm}$. The sample was placed in front of a $25 \mathrm{ps}$ pulsed beam produced by a $10 \mathrm{~Hz}$ repetition-rate parametric generator, tunable from 720 to $2100 \mathrm{~nm}$,

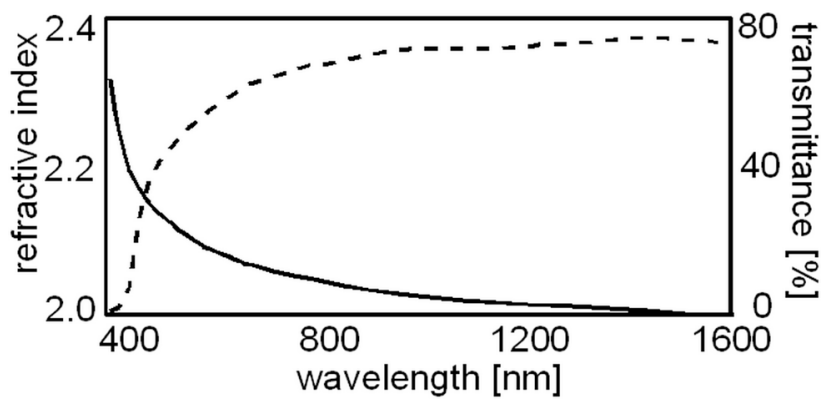

Fig. 1. NPG refractive index (solid line) and linear transmittance (dashes) versus wavelength. 


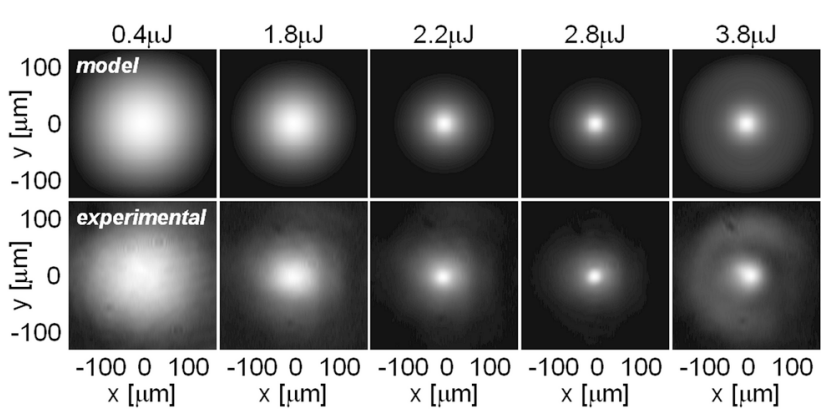

Fig. 2. Calculated (top) and observed (bottom) output profiles of an $11 \mu \mathrm{m}$ Gaussian beam propagating for $5.7 \mathrm{~mm}$ in NPG. The fitting parameters are $n_{2}=5.5 \times 10^{-15} \mathrm{~cm}^{2} / \mathrm{W}$ and $\beta_{3}=3 \times 10^{-4} \mathrm{~cm}^{3} / \mathrm{GW}^{2}$.

seeded by a parametric oscillator and synchronously pumped by an amplified frequency-doubled Nd:YAG laser. The beam, spatially filtered to a $\mathrm{TEM}_{00}$, was gently focused on the input facet by an $f=35 \mathrm{~mm}$ lens, employing polarizing optics to adjust both power and polarization. Photodetectors measured input and transmitted power, whereas a CCD camera and a microscope objective allowed us to collect images of the output beam profile. When excited at $1.064 \mu \mathrm{m}$, the NPG behaved as a pure Kerr medium; hence, it exhibited catastrophic collapse and optical damage visible at the output facet. Conversely, at a wavelength of $820 \mathrm{~nm}$ a beam with an $11 \mu \mathrm{m}$ waist could be launched at the input and collected at the output of the sample after propagating for $5.7 \mathrm{~mm}$. In this case, the output beam size (i.e., diffraction) decreased with excitation up to $2.8 \mu \mathrm{J}$, i.e., peak powers of $100 \mathrm{~kW}$, eventually generating a fundamental spatial soliton (solitary wave), as visible in Fig. 2. For excitations higher than $2.8 \mu \mathrm{J}$, a ring could be observed around the central spot (see the rightmost panel in the bottom row of Fig. 2). The latter ring is reminiscent of the transformation of a Gaussian into a Bessel beam in the presence of nonlinear losses [20]. Furthermore, at variance with previous observations at $1.064 \mu \mathrm{m}$, the sample did not undergo any damage for energies as high as $5.4 \mu \mathrm{J}$, as we verified by launching a weak probe (damage at the entrance facet was ascertained by microscope inspection for inputs $>5.4 \mu \mathrm{J})$. The different behavior at 1.064 and $0.82 \mu \mathrm{m}$ could be interpreted in terms of multiphoton absorption, as suggested by Fig. 3, where the measured transmittance of an $11 \mu \mathrm{m}$ beam is plotted versus pulse energy for propagation over a length of $11.2 \mathrm{~mm}$. The data, normalized to the linear transmittance (which accounts for Fresnel reflection), exhibit a progressive throughput reduction before permanent damage occurs.

Picosecond excitation allows us to exclude higherorder dynamics such as group velocity dispersion and plasma formation, which play a relevant role in fs filamentation $[12,13,16,23,24]$; the former is negligible, and the latter is sustained by avalanche ionization (versus multiphoton ionization at fs [25]), which leads to breakdown [26,27]. Considering that two and three photon energies at $820 \mathrm{~nm}$ are close to 3.02 and $4.54 \mathrm{eV}$ but absorption in amorphous materials can extend slightly below the bandgap, we resorted to simulations to identify the dominant contribution.

Light propagation in Kerr media is governed by the nonlinear Schrödinger equation:

$$
\begin{aligned}
& 2 i k \partial_{z} A+\nabla^{2} A+\frac{n_{2} k^{2}}{\eta_{0}}|A|^{2} A+i k \beta_{K}\left(\frac{n_{0}}{2 \eta_{0}}\right)^{K-1}|A|^{2 K-2} A \\
& \quad=0
\end{aligned}
$$

with $A$ being the slowly varying complex amplitude of the electric field $E(x, y, z, t)=1 / 2 A(x, y, z, t) \exp (i k z$ $-i \omega t)+$ cc, $k$ the wavenumber, $\eta_{0}$ the vacuum impedance, $n_{0}$ the refractive index, and $n_{2}$ the Kerr coefficient, as in $n(I)=n_{0}+n_{2} I(x, y, z)$, with $I$ the intensity. $\beta_{K}$ is the coefficient for $K$-photon absorption, as defined by $\partial_{z} I=-\beta_{K} I^{K}$.

To perform the numeric simulation we employed a $(2 \mathrm{D}+1)$ beam propagator with a standard CrankNicholson scheme and Gaussian excitation pulses. The best fits of the data (beam width and transmission at the output versus input energy) at $820 \mathrm{~nm}$ were obtained by setting $K=3$ in Eq. (1), yielding $n_{2}$ $=5.5 \times 10^{-15} \mathrm{~cm}^{2} / \mathrm{W}$ and $\beta_{3}=3 \times 10^{-4} \mathrm{~cm}^{3} / \mathrm{GW}^{2}$ for the cubic nonlinear index and the $3 \mathrm{PA}$ coefficient, respectively. Examples of time-integrated calculated profiles are shown in Fig. 2 (upper row). It should be emphasized that, while pointwise fits could be obtained using 2PA for given powers, 3PA reproduced the experimental results in a noncritical fashion, i.e., providing soliton stabilization in the whole available excitation range. This is apparent from the inset of Fig. 3 , displaying calculated peak intensities versus input energy for pure Kerr (dotted line), Kerr with 2PA (dashed line), and Kerr with 3PA (solid line) responses, respectively. The 2PA coefficients used are those that fit the output beam size as displayed in Fig. 2 for various energies; no stabilization was (numerically) obtained by 2PA for energies exceeding $3.8 \mu \mathrm{J}$ and the same input waist.

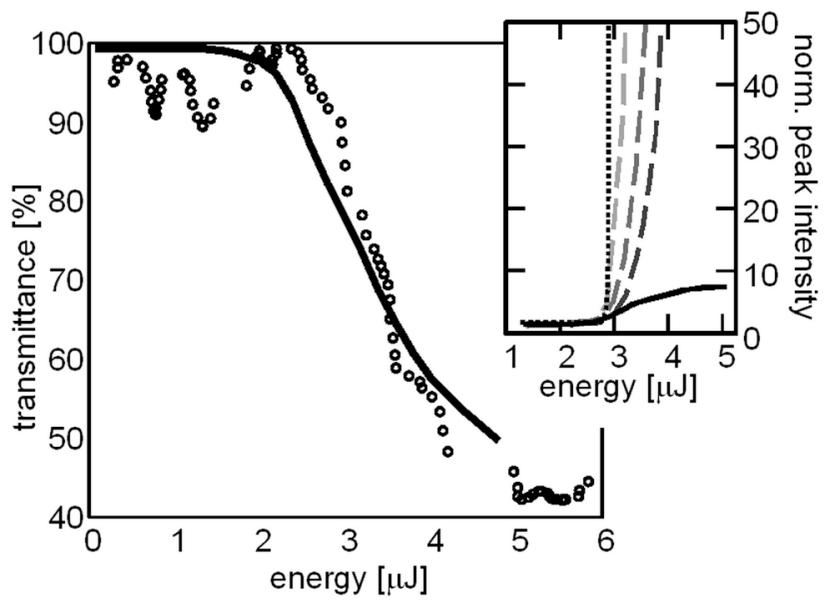

Fig. 3. Transmittance at $0.82 \mu \mathrm{m}$ versus excitation: data (dots) and simulation (solid line) from Eq. (1) with parameters of Fig. 2. The inset shows calculations of the (input normalized) peak intensity when considering 2PA (dashed lines for $0.012,0.018$, and $0.024 \mathrm{~cm} / \mathrm{GW}$, left to right) or $3 \mathrm{PA}$ (solid line, $3 \times 10^{-4} \mathrm{~cm}^{3} / \mathrm{GW}^{2}$ ), compared with the pure Kerr case (dots). 


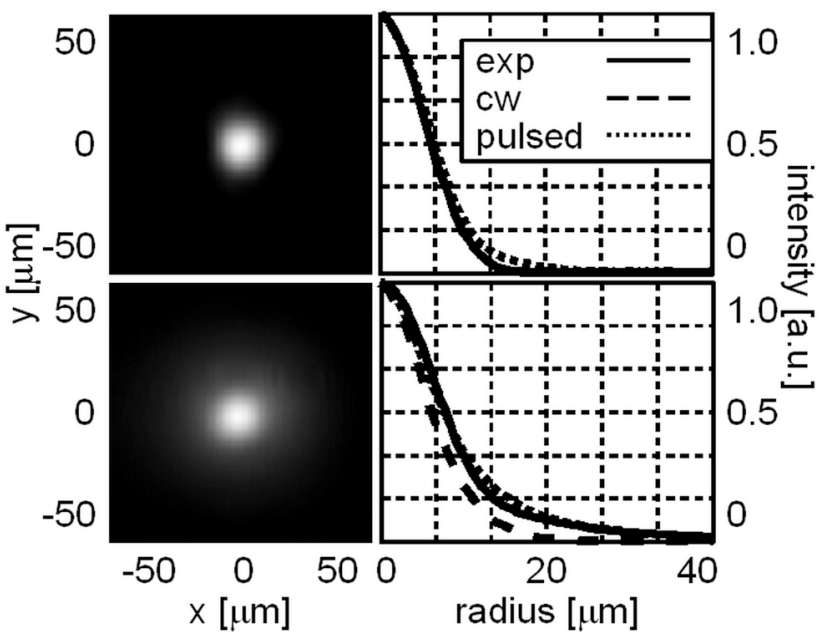

Fig. 4. (Top) Input and (bottom) output beam profiles for propagation over $5.7 \mathrm{~mm}$ and input energy $2.8 \mu \mathrm{J}$ (peak $100 \mathrm{~kW}$ ): photos (left) and graphs of the radial intensity (right, thick lines). The dotted (dashed) lines are calculated profiles after integration over time (for $\mathrm{cw}$ excitation at the peak).

These simulations indicate that 3PA absorption at $820 \mathrm{~nm}$ can stabilize the beam into a spatial soliton: self-confined beams could propagate over several (>6) Rayleigh lengths without damaging the sample. Figure 4 displays photos and simulations of the beam profile at input and output facets after propagation for $5.7 \mathrm{~mm}$, for input energy of $2.8 \mu \mathrm{J}$ (peak power $100 \mathrm{~kW}$ ), and nonlinear absorption amounting to about $20 \%$.

In conclusion, we experimentally investigated nonlinear propagation of 25 ps pulsed Gaussian beams in a heavy metal oxide glass of the ternary system $\mathrm{Nb}_{2} \mathrm{O}_{5} \mathrm{GeO}_{2} \mathrm{PbO}$. The results at $\lambda=820 \mathrm{~nm}$ indicate that a near-resonant 3PA stabilizes the Kerr response, yielding spatial solitons that are stable over more than six diffraction lengths. The cubic ultrafast nonlinearity of NPG is promising for soliton-based interconnects and all-optical switching.

This work was funded by an "Integrated Action" between Italy and Spain (IT1890/HI2006-0095) and by the Spanish Ministry of Education and Science (MAT2005-06508-C02-01 and TEC2005-00074/MIC). The authors thank J. M. Fernandez-Navarro (Istituto de Optica) for the samples. S. Stivala acknowledges support for his Ph.D. from the Department of Electronic, Electrical and Telecommunication Engineering at the University of Palermo.

\section{References}

1. R. Y. Chiao, E. Garmire, and C. H. Townes, Phys. Rev. Lett. 13, 479 (1964).
2. G. I. Stegeman and M. Segev, Science 286, 1518 (1999).

3. Y. S. Kivshar and G. P. Agrawal, Optical Solitons (Academic, 2003).

4. G. Assanto and G. I. Stegeman, Opt. Express 39, 13 (2003).

5. C. Conti and G. Assanto, in Encyclopedia of Modern Optics, R. D. Guenther, D. G. Steel, and L. Bayvel, eds. (Elsevier, 2004), Vol. 5, pp. 43-55.

6. G. Assanto and M. Peccianti, IEEE J. Quantum Electron 39, 13 (2003).

7. M. K. T. Clement, J. S. Hayden, Y. T. Hayden, H.-J. Hoffman, F. T. Lentes, and N. Neuroth, in The Properties of Optical Glass, M. Bach and N. Neuroth, eds. (Springer-Verlag, 1995), pp. 19-165.

8. M. E. Lines, J. Appl. Phys. 69, 6876 (1991).

9. J. M. Harbold, F. O. Ilday, F. W. Wise, J. S. Sanghera, V. Q. Nguyen, L. B. Shaw, and I. D. Aggarwal, Opt. Lett. 27, 119 (2002).

10. J. S. Aitchison, A. M. Weiner, Y. Silberberg, M. K. Oliver, J. L. Jackel, D. E. Leaird, E. M. Vogel, and P. W. E. Smith, Opt. Lett. 15, 471 (1990).

11. H. S. Eisenberg, R. Morandotti, Y. Silberberg, S. BarAd, D. Ross, and J. S. Aitchison, Phys. Rev. Lett. 87, 043902 (2001).

12. S. Tzortzakis, L. Sudrie, M. Franco, B. Prade, A. Mysyrowicz, A. Couairon, and L. Bergé, Phys. Rev. Lett. 87, 213902 (2001).

13. L. Sudrie, A. Couairon, M. Franco, B. Lamouroux, B. Prade, S. Tzortzakis, and A. Mysyrowicz, Phys. Rev. Lett. 89, 186601 (2002).

14. C. Rotschild, O. Cohen, O. Manela, M. Segev, and T. Carmon, Phys. Rev. Lett. 95, 213904 (2005).

15. Z. Chen, M. Asaro, O. Ostroverkhova, W. E. Moerner, M. He, and R. J. Twieg, Opt. Lett. 28, 2509 (2003).

16. S. Tzortzakis, D. G. Papazoglou, and I. Zergioti, Opt. Lett. 31, 796 (2006).

17. W. H. Dumbaugh and J. C. Lapp, J. Am. Ceram. Soc. 75, 2315 (1992).

18. C. B. de Araùjo, E. L. Falcao-Filho, A. Humeau, D. Guichaoua, G. Boudebs, and L. R. P. Kassab, Appl. Phys. Lett. 87, 221904 (2005).

19. I. Kang, T. D. Krauss, F. W. Wise, B. G. Aitken, and N. F. Borrelli, J. Opt. Soc. Am. B 12, 2053 (1995).

20. A. Dubietis, E. Gaizauskas, G. Tamosauskas, and P. Di Trapani, Phys. Rev. Lett. 92, 253903 (2004).

21. J. Gonzalo, O. Sanz, A. Perea, J. M. FernandezNavarro, C. N. Afonso, and J. Garcìa-Lòpez, Appl. Phys. A 76, 943 (2003).

22. R. Balda, L. M. Lacha, J. Fernandez, and J. M. Fernandez-Navarro, Opt. Mater. 27, 1771 (2005).

23. S. Skupin, R. Nuter, and L. Bergé, Phys. Rev. A 74, 043813 (2006).

24. A. Couairon, E. Gaizauskas, D. Faccio, A. Dubietis, and P. Di Trapani, Phys. Rev. E 73, 016608 (2006).

25. B. C. Stuard, M. D. Feit, A. M. Rubenchik, B. W. Shore, and M. D. Perry, Phys. Rev. Lett. 74, 2248 (1995).

26. N. Bloembergen, IEEE J. Quantum Electron. 10, 375 (1974).

27. E. Yablontovic and N. Bloembergen, Phys. Rev. Lett. 29, 907 (1972). 